\title{
Flebotomíneos (Diptera, Psychodidae) em reservas florestais da área metropolitana de São Luís, Maranhão, Brasil
}

\author{
Robert Menezes Marinho ${ }^{1}$, Raquel Silva Fonteles², Gabriel Costa e Vasconcelos², Patrícia Castelo Branco \\ Azevêdo ${ }^{2}$, Jorge Luiz Pinto Moraes ${ }^{4} \&$ José Manuel Macário Rebêlo $^{3,4}$
}

${ }^{1}$ Egresso do Mestrado em Saúde e Ambiente, Universidade Federal do Maranhão. Praça Madre Deus, no 02, 65.025-560 Bairro Madre Deus, São Luís-MA, Brasil

${ }^{2}$ Egresso do Mestrado em Biodiversidade e Conservação. Departamento de Biologia, Universidade Federal do Maranhão, Avenida dos Portugueses S/N Campus do Bacanga, São Luís-MA.

${ }^{3}$ Departamento de Biologia, Universidade Federal do Maranhão, Avenida dos Portugueses S/N Campus do Bacanga, São Luís-MA.

${ }^{4}$ Laboratório de Entomologia e Vetores. Departamento de Patologia, Universidade Federal do Maranhão. Praça Madre Deus n 2, 65025-560, São Luís-MA, Brasil. macariorebelo@uol.com.br

\begin{abstract}
Phlebotomines (Diptera, Psychodidae) in forest reserves in the metropolitan area of São Luís, Maranhão, Brazil. This study deals with the diversity, relative abundance and seasonality of sandflies species in two modified primary forest fragments, situated at São Luís city, capital of the state of Maranhão. The sandflies were captured with the use of light traps, CDC (Center Control Disease) type, installed at the edge and in the center of the forest, at the height of 1.5 meter, being there from 6 p.m. up to 6 a.m., once per month, during a year time. An amount of 1,356 specimens from 23 species, which 21 belonged to genera Lutzomyia and two belonged to genera Brumptomyia, were captured. In the Sacavém forest reserve, 1,061 specimens were captured, resulting in a capture effort of 2.5 individuals/ hour/trap, most of them L. longipalpis (44.8\%), followed by L. antunesi (36.4\%), L. sordelli (5.9\%), L. flaviscutellata (3.9\%) and L. whitmani $(2.1 \%)$. The highest concentrations of species (11) and individuals $(60.1 \%)$ occurred during the rainy period. In the Itaqui forest reserve, 295 specimens were collected and the capture effort was 0.2 individuals/hour/ trap, most of them were L. flaviscutellata, $(58.6 \%)$, L. sordelli $(14.6 \%)$, L. longipalpis $(7.1 \%)$, L. evandroi $(6.4 \%), L$. longipennis $(3 \%)$, L. trinidadensis $(2.7 \%)$ and L. whitmani $(1.7 \%)$. The species were more frequent during the dry season (11) and specimens during the rainy spell (54.6\%).
\end{abstract}

KEYWORDS. Amazonia; Ecology; Forest fragments.

RESUMO. Flebotomíneos (Diptera, Psychodidae) em reservas florestais da área metropolitana de São Luís, Maranhão, Brasil. Estudou-se a diversidade, a abundância relativa e a sazonalidade das espécies de flebotomíneos em duas reservas de floresta primária alterada, a reserva florestal do Sacavém (RFS) e reserva florestal do Itaqui (RFI), localizadas na área metropolitana de São Luís, capital do estado do Maranhão. Os flebotomíneos foram capturados com armadilhas luminosas tipo CDC, instaladas na borda e no centro da mata, a 1,5 metros de altura, das $18 \mathrm{~h} 00$ às $6 \mathrm{~h} 00$, uma vez por mês, durante um ano. No total foram capturados 1.356 indivíduos de 23 espécies dos gêneros Lutzomyia (21) e Brumptomyia (2). Na RFS foram capturados 1.061 espécimes, resultando num esforço de captura de 2,5 indivíduos/hora/armadilha e o predomínio de L. longipalpis $(44,8 \%)$, seguida por L. antunesi $(36,4 \%)$, L. sordelli $(5,9 \%)$, L. flaviscutellata $(3,9 \%)$ e L. whitmani $(2,1 \%)$. O maior número de espécies (11) e indivíduos $(60,1 \%)$ ocorreu na estação chuvosa. Na RFI foram obtidos 295 espécimes, o esforço de captura foi 0,2 indivíduos/hora/armadilha, com o predomínio de L. flaviscutellata $(58,6 \%), L$. sordelli $(14,6 \%)$, L. longipalpis $(7,1 \%)$, L. evandroi $(6,4 \%)$, L. longipennis $(3 \%)$, L. trinidadensis $(2,7 \%)$ e L. whitmani $(1,7 \%)$. As espécies foram mais numerosas na estiagem (11) e os espécimes durante a estação chuvosa $(54,6 \%)$.

PALAVRAS-CHAVE. Amazônia; Ecologia; Fragmento de Floresta.

Os flebotomíneos são naturalmente diversificados nas florestas tropicais úmidas (Ward et al. 1973, mas muitas espécies vêm invadindo os ambientes peridomiciliares de zonas rurais e urbanas em diversas áreas endêmicas para leishmanioses no Brasil. A capacidade de freqüentar ambientes artificiais e a ocorrência em alta densidade são condições que incriminam os flebotomíneos como vetores das leishmanioses (Gomes \& Galati 1989).

No Estado do Maranhão, são conhecidas mais de 30 espécies de flebotomíneos que se associam com áreas florestais (Rebêlo et al. 2000a, b) próximas a povoados rurais, onde são muito freqüentes nos ambientes peridomiciliares.
Na ilha de São Luís, já foram encontradas 28 espécies associadas com áreas de floresta secundária (capoeira) ou ambientes peridomiciliares (Rebêlo et al. 1999a), não se dispondo, ainda, de dados de áreas florestais primárias.

A realização de levantamento da fauna de flebotomíneos em ecossistemas naturais é importante para ampliar o conhecimento das áreas de ocorrência desses insetos, subsidiar projetos conservacionistas e minimizar o contacto deles com o homem. Além disso, a compreensão da dinâmica populacional destes insetos pode se revelar como um importante fator para a implantação de políticas de controle epidemiológico e de manejo florestal. 
Em áreas consideradas endêmicas para leishmaniose há vários estudos sobre flebotomíneos silvestres, como no município de Buriticupu, na Amazônia do Maranhão (Rebêlo et al. 2000a b), contudo, em áreas florestais do entorno do município de São Luís há carência desses estudos. Nesse sentido, estudou-se a fauna de flebotomíneos de duas reservas de floresta primária alterada, localizadas na área metropolitana de São Luís, tendo como objetivos conhecer a diversidade, abundância relativa e a sazonalidade das espécies.

\section{MATERIALE MÉTODOS}

Área de estudo. $\mathrm{O}$ estudo foi realizado em duas reservas de formação florestal latifoliada (remanescentes da floresta amazônica), na periferia da área metropolitana de São Luís, localizado, entre $02^{\circ} 28^{\prime} 12^{\prime \prime}$ e $02^{\circ} 48^{\prime} 09^{\prime \prime} \mathrm{Se} 44^{\circ} 10^{\prime} 18^{\prime \prime}$ e $44^{\circ} 35^{\prime}$ 37" W a 24 metros de altitude com $831,7 \mathrm{Km}^{2}$. 1) a reserva florestal do Itaqui (RFI), com uma área de $3,5 \mathrm{~km}^{2}$, pertence à Companhia Vale do Rio Doce, 2) a reserva florestal do Sacavém (RFS), com uma área de 5,6 km², pertence à Companhia de Água e Esgoto do Maranhão.

A cobertura vegetal original do município é um misto de floresta latifoliada, babaçual, vegetação de dunas/restinga e manguezal. Contudo, a expansão das áreas industrializadas, o desmatamento para fins de urbanização, o sistema de corte e queimadas utilizado no cultivo, a extração mineral de areia, argila, laterita, e madeira nas zonas de capoeira vêm reduzindo sensivelmente as áreas florestadas e degradando os solos (IPPC 2006). As áreas florestais da Ilha restringem-se hoje a pequenas áreas de proteção ambiental.

Coletas. Para a captura de flebotomíneos foram usadas 13 armadilhas luminosas tipo CDC, instaladas na borda e no centro da mata, a 1,5 $\mathrm{m}$ de altura. Foram utilizadas dez armadilhas na RFI, a 50 m uma da outra e três na RFS, a 500 m entre si. Cada armadilha funcionou 12 horas ininterruptamente, das 18:00 às 6:00 horas, uma vez por mês, durante um ano, totalizando um esforço de captura de 144 horas por mês.

Os insetos capturados foram transportados para o Laboratório de Entomologia e Vetores da Universidade Federal do Maranhão, acondicionados em freezer e, posteriormente, submetidos à triagem sob estereomicroscópio para a separação dos flebotomíneos. Os espécimes triados foram montados entre lâminas e lamínulas e identificados com auxílio das chaves dicotômicas de Young \& Duncan (1994).

Análise dos dados. Para análise das diferenças nas proporções de indivíduos capturados entre os ambientes e estações utilizou-se o teste Qui-Quadrado $\left(\chi^{2}\right)$. A análise da abundância relativa das espécies predominantes e seus limites de confiança foram calculados, utilizando o método descrito em Laroca (1995). Foi aplicada a correlação não-paramétrica de Spearman entre o número de espécimes e as médias da precipitação pluviométrica, medidos ao longo do ano. Calculouse também o índice de Constância dos flebotomíneos (Silveira Neto et al. 1976).

\section{RESULTADOS}

Foram capturadas 23 espécies de flebotomíneos, sendo 15 na RFS e igual número na RFI, com sete espécies compartilhando as duas reservas (Tab. I), assim distribuídas, duas espécies do gênero Brumptomyia: B. avellari Costa Lima e B. travassoi Mangabeira; e vinte uma do gênero Lutzomyia: L. anduzei Rozeboom, L. antunesi Coutinho, L. carrerai carrerai Barret, L. claustrei Abonnenc, Léger \& Fauran, L. evandroi Costa Lima \& Antunes, L. flaviscutellata Mangabeira, L. furcata Mangabeira, L. infraspinosa Mangabeira, L. longipalpis Lutz \& Neiva, L. longipennis Barreto, L. olmeca nociva Young \& Arias, L. oswaldoi Mangabeira, L. paraensis Forattini, L. richardwardi Ready \& Fraiha, L. rorotaensis Floch \& Abonnenc, L. serrana Damasceno \& Arouck, L. servulolimai Damasceno \& Causey, L. shawi Fraiha, Ward \& Ready, L. sordelli Shannon \& Del Pont, L. trinidadensis Newstead e L. whitmani Antunes \& Coutinho. Do total, foram capturados 1.356 espécimes distribuídos na RFS $(78,2 \%)$ e RFI $(21,8 \%)$ e a freqüência de fêmeas foi maior do que a de machos (RFS, 56,6\% e RFI, $63,1 \%)$.

Na RFS foram capturados 1.061 espécimes, resultando num esforço de captura de 2,5 indivíduos/hora/armadilha. As espécies predominantes foram L. longipalpis $(44,8 \%)$, seguida por L. antunesi $(36,4 \%), L$. sordelli (5,9\%), L. flaviscutellata $(3,9 \%)$ e L. whitmani $(2,1 \%)$.

Na RFI coletou-se apenas 295 espécimes, resultando num esforço de captura de 0,2 indivíduos/hora/armadilha. $\mathrm{O}$ predomínio foi de $L$. flaviscutellata $(58,6 \%)$, seguida por $L$. sordelli (14,6\%), L. longipalpis (7,1\%), L. evandroi (6,4\%), $L$. longipennis (3\%), L. trinidadensis $(2,7 \%)$ e L. whitmani $(1,7 \%)$.

$\mathrm{Na}$ RFS apenas L. longipalpis foi capturada em todos os meses do ano e $L$. antunesi só não foi capturada em janeiro (Tab. II). L. longipalpis, L. antunesi, L. flaviscutellata e $L$. whitmani estiveram presentes em mais de $50 \%$ das coletas, $L$. claustrei, L. evandroi, L. olmeca nociva e L. paraensis ocorreram entre $25 \%$ e $50 \%$ das coletas, enquanto $L$. anduzei, L. carrerai, $L$. furcata, $L$. oswaldoi, $L$. serrana e B. travassoi estavam representadas em menos de $25 \%$ das coletas. A maior concentração de flebotomíneos foi registrada na estação chuvosa $(60,1 \%)$, e em cada estação foram coletadas 12 espécies. Não houve correlação entre a variação mensal dos valores médios da umidade relativa do $\operatorname{ar}(\mathrm{r}=-0,06, \mathrm{p}=0,85) \mathrm{e}$ da temperatura $(r=-0,07 ; p=0,84)$, bem como, dos valores absolutos da precipitação pluviométrica $(r=0,16 ; p=0,62)$ e a variação nos números de espécimes de flebotomíneos coletados ao longo do ano.

Na RFI, L. flaviscutellata e $L$. sordellii ocorreram em 11 e 10 meses do ano, respectivamente e em conjunto com $L$. evandroi, L. longipalpis, L. longipennis estavam presentes em mais de $50 \%$ das coletas, $L$. trinidadensis e $L$. whitmani, entre $25 \%$ e $50 \%$, e B. avellari, L. infraspinosa, L. oswaldoi, $L$. richardwardi, L. rorotaensis, $L$. servulolimai e $L$. shawi, em menos de $25 \%$ (Tab. III). Os flebotomíneos estiveram presentes o ano todo, com número semelhante de espécies presentes em 
Tabela I. Flebotomíneos capturados na Reserva Florestal de Sacavém (RFS), de agosto de 2001 a julho de 2002, e Reserva Florestal de Itaqui (RFI), de março de 2005 a fevereiro de 2006, na área metropolitana de São Luís, Maranhão, Brasil.

\begin{tabular}{|c|c|c|c|c|c|c|c|c|c|c|}
\hline \multirow{2}{*}{$\begin{array}{l}\text { Locais } \\
\text { Espécie / Sexo }\end{array}$} & \multicolumn{3}{|c|}{ RFS } & \multicolumn{3}{|c|}{ RFI } & \multicolumn{3}{|c|}{ Soma } & \multirow[b]{2}{*}{$\%$} \\
\hline & M & F & Total & M & F & Total & M & $\mathrm{F}$ & $n^{\circ}$ & \\
\hline B. avellari & - & - & - & 2 & - & 2 & - & - & 2 & 0,1 \\
\hline B. travassoi & 2 & - & 2 & - & - & - & 2 & - & 2 & 0,1 \\
\hline L. anduzei & 2 & - & 2 & - & - & - & 2 & - & 2 & 0,1 \\
\hline L. antunesi & 385 & 1 & 386 & - & - & - & 385 & 1 & 386 & 28,5 \\
\hline L. carrerai & - & 2 & 2 & - & - & - & & 2 & 2 & 0,1 \\
\hline L. claustrei & 19 & - & 19 & 1 & 1 & 2 & 20 & 1 & 21 & 1,6 \\
\hline L. evandroi & 2 & 4 & 6 & 12 & 7 & 19 & 14 & 11 & 25 & 2,0 \\
\hline L. flaviscutellata & 24 & 17 & 41 & 48 & 125 & 173 & 72 & 142 & 214 & 15,8 \\
\hline L. furcata & 2 & - & 2 & & & & 2 & - & 2 & 0,1 \\
\hline L. infraspinosa & - & - & - & - & 1 & 1 & & 1 & 1 & 0,1 \\
\hline L. longipalpis & 8 & 467 & 475 & 6 & 15 & 21 & 14 & 482 & 496 & 36,6 \\
\hline L. longipennis & - & - & - & 6 & 3 & 9 & 6 & 3 & 9 & 0,7 \\
\hline L. olmeca nociva & 3 & 18 & 21 & - & - & - & 3 & 18 & 21 & 1,6 \\
\hline L. oswaldoi & 5 & - & 5 & 1 & - & 1 & 6 & - & 6 & 0,4 \\
\hline L. paraensis & 6 & 8 & 14 & - & - & - & 6 & 8 & 14 & 1,0 \\
\hline L. richardwardi & - & - & - & 2 & - & 2 & 2 & - & 2 & 0,1 \\
\hline L. rorotaensis & - & - & - & 3 & - & 3 & 3 & - & 3 & 0,2 \\
\hline L. serrana & 1 & - & 1 & - & - & - & 1 & - & 1 & 0,1 \\
\hline L. servulolimai & - & - & - & 2 & - & 2 & 2 & - & 2 & 0,1 \\
\hline L. shawi & - & - & - & 3 & 1 & 4 & 3 & 1 & 4 & 0,3 \\
\hline L. sordelli & - & 63 & 63 & 16 & 27 & 43 & 16 & 90 & 106 & 7,8 \\
\hline L. trinidadensis & - & - & - & 5 & 3 & 8 & 5 & 3 & 8 & 0,6 \\
\hline L. whitmani & 2 & 20 & 22 & 2 & 3 & 5 & 2 & & 27 & 2,0 \\
\hline Total & 461 & 600 & 1.061 & 109 & 186 & 295 & 570 & 786 & 1.356 & 100,0 \\
\hline$\%$ & 43,4 & 56,6 & 78,3 & 36,9 & 63,1 & 21,7 & 42,0 & 58,0 & 100,0 & \\
\hline Número de espécies & & & 15 & & & 15 & & & 23 & \\
\hline
\end{tabular}

B.=Brumptomyia $;$ L. $=$ Lutzomyia $\mathrm{M}=$ machos $; \mathrm{F}=$ fêmeas.

ambas as estações e maior abundância de indivíduos na estação chuvosa (54,6\%). Não houve correlação entre a variação dos espécimes distribuídos ao longo do ano com os valores da precipitação pluviométrica $(r=0,22 ; \mathrm{p}=0,50)$, temperatura $(\mathrm{r}=-0,55 ; \mathrm{p}=0,06)$ e umidade relativa do $\operatorname{ar}(\mathrm{r}=$ $0,31, \mathrm{p}=0,32)$.

\section{DISCUSSÃO}

A riqueza de espécies de flebotomíneos foi elevada e equivalente nas duas reservas florestais, quando comparada à riqueza de área de capoeira (Barros et al. 2000) dos ambientes peridomiciliar de áreas rurais (Carvalho et al. 2000) e urbanas (Araújo et al. 2000) da ilha de São Luís.

Dentre as espécies capturadas, $78 \%$ ainda não foram encontradas em áreas rurais (Carvalho et al. 2000) e urbanas (Araújo et al. 2000), comportando-se como espécies estritamente silvestres, como é o caso de B. avellari, $B$. travassoi, L. anduzei, L. carrerai, L. claustrei, L. furcata, $L$. infraspinosa, L. longipennis, L. olmeca nociva, L. oswaldoi, $L$. paraensis, $L$. richardwardi, $L$. rorotaensis, $L$. serrana, $L$. servulolimai, L. shawi, L. sordelli, L. trinidadensis.

Contudo, nem todas as espécies restringem o seu domínio de ocorrência às áreas florestais. L. evandroi e L. longipalpis são freqüentes em invasões e outras formas desordenadas de ocupação humana nos municípios da ilha de São Luís, incluindo Raposa (Araújo et al. 2000), São José de Ribamar (Carvalho et al. 2000), bem como em área de vegetação arbórea de pequeno porte, como no Paço do Lumiar (Barros et al. 2000). A última é considerada uma espécie constante, com elevado potencial biótico e resistência às estratégias de controle vetorial em áreas endêmicas de calazar (Costa et al. 1990). L. antunesi e $L$. flaviscutellata são comuns em áreas florestais (Shaw \& Lainson 1972, Ward et al. 1973), mas podem ocorrer em capoeiras, bosques, plantações e áreas antropogências (Ryan et al. 1987). L. whitmani vem se adaptando ao peridomicílio de algumas cidades com foco de transmissão de leishmaniose tegumentar, como Codó (Rebêlo et al. 1999b) e D. Pedro (Leonardo \& Rebêlo 2004).

A ocorrência de algumas espécies de flebotomíneos em áreas urbanas deve-se à capacidade desses insetos se adaptarem onde houve profundas mudanças nos seus habitats naturais (florestas). A tendência dos flebotomíneos de invadirem o ambiente antrópico, tem sido constatada em áreas das regiões Norte (Feitosa \& Castellón 2004), devido a alterações de floresta, que provocaram a restrição de espaços ecológicos. Considerando a importância dos flebotomíneos e a localização da RFS e RFI nas proximidades da área metropolitana de São Luís, é aconselhável manter contínua vigilância na zona de entorno das referidas reservas. 
Tabela II. Sazonalidade de flebotomíneos capturados na Reserva Florestal de Sacavém, na área metropolitana de São Luís, Maranhão, Brasil, de agosto de 2001 a julho de 2002.

\begin{tabular}{|c|c|c|c|c|c|c|c|c|c|c|c|c|}
\hline \multirow{2}{*}{$\begin{array}{l}\text { Estação } \\
\text { Espécie/Mês }\end{array}$} & \multicolumn{6}{|c|}{ Chuvosa } & \multicolumn{6}{|c|}{ Seca } \\
\hline & $\mathrm{J}$ & $\mathrm{F}$ & $\mathrm{M}$ & A & M & $\mathrm{J}$ & $\mathrm{J}$ & A & $\mathrm{S}$ & $\mathrm{O}$ & $\mathrm{N}$ & $\mathrm{D}$ \\
\hline B. travassoi & - & - & 1 & - & - & - & - & 01 & - & - & - & - \\
\hline L. anduzei & - & 2 & - & - & - & - & - & - & - & - & - & - \\
\hline L. antunesi & - & 217 & 3 & 1 & 6 & 29 & 5 & 68 & 37 & 15 & 3 & 2 \\
\hline L. carrerai & - & - & - & - & - & - & 2 & - & - & - & - & - \\
\hline L. claustrei & - & 1 & 1 & - & 7 & - & 8 & 1 & 1 & - & - & - \\
\hline L. evandroi & 1 & - & 1 & 1 & 1 & - & - & 1 & - & - & 1 & - \\
\hline L. flaviscutellata & - & 20 & - & 2 & 4 & - & 6 & 3 & 5 & 1 & - & - \\
\hline L. furcata & - & - & - & - & - & - & - & 2 & - & - & - & - \\
\hline L. longipalpis & 119 & 49 & 13 & 6 & 5 & 32 & 46 & 130 & 19 & 35 & 16 & 5 \\
\hline L. olmeca nociva & - & 14 & - & 4 & - & 3 & - & - & - & - & - & - \\
\hline L. oswaldoi & - & 4 & - & 1 & - & - & - & - & - & - & - & - \\
\hline L. paraensis & - & 4 & 3 & 2 & 1 & 3 & - & - & 1 & - & - & - \\
\hline L. serrana & - & - & - & - & - & - & - & - & - & - & 1 & - \\
\hline L. sordelli & 5 & 38 & 11 & 1 & 3 & - & 3 & 1 & 1 & - & - & - \\
\hline L. whitmani & 1 & 7 & 3 & 1 & 4 & 3 & 2 & - & 1 & - & - & - \\
\hline Total & 126 & 356 & 36 & 19 & 31 & 70 & 72 & 207 & 65 & 51 & 21 & 7 \\
\hline Número de espécies & 5 & 11 & 8 & 10 & 9 & 6 & 8 & 9 & 8 & 4 & 5 & 3 \\
\hline Umidade relativa \% & 86,6 & 83,9 & 88,0 & 90,3 & 87,0 & 87,0 & 83,0 & 79,4 & 75,5 & 76,2 & 76,8 & 77,9 \\
\hline Temperatura $\left({ }^{\circ} \mathrm{C}\right)$ & 27,5 & 28,4 & 27,6 & 27,2 & 28,0 & 27,6 & 28,3 & 29,0 & 29,1 & 29,5 & 29,0 & 29,2 \\
\hline Pluviosidade (mm) & 479 & 109 & 344 & 3,2 & 231 & 205 & 60 & 0 & 7,9 & 0 & 0,2 & 42,8 \\
\hline
\end{tabular}

B.=brumptomyia $;$ L $=$ Lutzomyia $; \mathrm{M}=$ machos; $\mathrm{F}=$ fêmeas.

A presença de L. longipalpis, L. flaviscutellata e L. whitmani é motivo de alerta para a possibilidade de surtos de leishmaniose visceral e tegumentar nos bairros periféricos da área metropolitana de São Luís, em função de desequilíbrios causados por modificações antrópicas nos habitats dos flebotomíneos.

A sazonalidade das espécies não diferiu do padrão que tem sido encontrado em outras áreas da ilha de São Luís (Rebêlo 2001). Primeiro, pela presença de muitas espécies acessórias (L. claustrei, L. evandroi, L. olmeca nociva, L. paraensis, L. trinidadensis e $L$. whitmani) e acidentais ( $L$. anduzei, L. carrerai, L. furcata, L. infraspinosa, L. oswaldoi, L. richardwardi, L. rorotaensis, L. serrana, L. servulolimai, L. shawi e B. avellari e B. travassoi) se sucedendo ao longo

Tabela III. Sazonalidade de flebotomíneos capturados na Reserva Florestal de Itaqui, na área metropolitana de São Luís, Maranhão, Brasil, de março de 2005 a fevereiro de 2006.

\begin{tabular}{|c|c|c|c|c|c|c|c|c|c|c|c|c|}
\hline \multirow{2}{*}{$\begin{array}{l}\text { Estação } \\
\text { Espécie/Mês }\end{array}$} & \multicolumn{6}{|c|}{ Chuvosa } & \multicolumn{6}{|c|}{ Seca } \\
\hline & $\mathrm{J}$ & $\mathrm{F}$ & $\mathrm{M}$ & A & M & $\mathrm{J}$ & $\mathrm{J}$ & A & $\mathrm{S}$ & $\mathrm{O}$ & $\mathrm{N}$ & $\mathrm{D}$ \\
\hline B. avellari & - & - & - & - & - & - & - & - & 2 & - & - & - \\
\hline L. evandroi & 1 & 2 & 1 & 1 & - & - & - & - & 4 & 6 & 1 & 3 \\
\hline L. flaviscutellata & 4 & 11 & - & 55 & 25 & 24 & 24 & 13 & 10 & 2 & 3 & 2 \\
\hline L. infraspinosa & - & - & - & - & - & 1 & - & - & - & - & - & - \\
\hline L. longipalpis & - & - & 2 & 2 & - & 1 & - & - & 3 & 4 & 4 & 5 \\
\hline L. longipennis & 2 & 1 & 1 & 1 & - & - & 2 & - & 1 & 1 & - & - \\
\hline L. oswaldoi & - & - & 1 & - & - & - & - & - & - & - & - & - \\
\hline L. richardwari & - & - & - & - & - & - & 1 & - & 1 & - & - & - \\
\hline L. rorotaensis & 2 & - & 1 & - & - & - & - & - & - & - & - & - \\
\hline L. servulolimai & - & - & - & 1 & - & - & - & - & 1 & - & - & - \\
\hline L. sordelli & 1 & 1 & 6 & 4 & - & 1 & 6 & 3 & 12 & 8 & - & 1 \\
\hline L. trinidadensis & - & - & 2 & 4 & - & 1 & 1 & - & - & - & - & - \\
\hline L. whitmani & - & - & - & - & - & - & 1 & - & - & - & 3 & 1 \\
\hline L. shawi & - & - & - & - & - & - & - & - & - & - & 2 & 2 \\
\hline Total & 10 & 15 & 14 & 68 & 25 & 28 & 35 & 16 & 34 & 21 & 13 & 14 \\
\hline Número de Espécies & 5 & 4 & 7 & 7 & 1 & 5 & 6 & 2 & 8 & 5 & 5 & 6 \\
\hline Umidade relativa \% & 75,6 & 78,4 & 80,8 & 81,6 & 79,7 & 79,2 & 76,8 & 72 & 68,3 & 66,3 & 67,4 & 70,4 \\
\hline Temperatura $\left({ }^{\circ} \mathrm{C}\right)$ & 23,8 & 23,7 & 23,9 & 23,5 & 23,3 & 22,9 & 22,5 & 23,5 & 24,6 & 25,2 & 25,3 & 24,6 \\
\hline Pluviosidade (mm) & 222,3 & 224,3 & 352 & 421,2 & 546,4 & 134,5 & 93,4 & 50,4 & 68,9 & 1,8 & 6,5 & 60 \\
\hline
\end{tabular}


do ano, ao lado de poucas que se comportam como constantes (L. antunesi, L. flaviscutellata, L. evandroi L. longipalpis, longipennis, L. sordellii e L. whitmani), resultando em um considerável número de espécies presentes em todos os meses do ano; segundo, pela maior freqüência de flebotomíneos na estação chuvosa.

Em síntese, esse estudo mostrou a existência de uma fauna de flebotomíneos diversificada, com oito espécies que ainda não tinham registro de ocorrência na ilha de São Luís, maior abundância no período chuvoso e predomínio de espécies acessórias e acidentais.

Agradecimentos. Á Companhia de Água e Esgoto do Maranhão e Companhia Vale do Rio Doce por terem permitido a execução do trabalho na Reserva Florestal do Sacavém e Reserva Florestal do Itaqui, respectivamente; à estação meteorológica da Universidade Estadual do Maranhão; ao CNPq pelo apoio financeiro, à CAPES e a FAPEMA pelas bolsas de Mestrado e de Iniciação Científica.

\section{REFERÊNCIAS}

Araújo, J. C; J. M. M. Rebêlo; M. L. Carvalho \& V. L. L. Barros. 2000. Composição dos flebotomíneos (Diptera, Psychodidae) do município da Raposa-MA, Brasil. Área endêmica de leishmanioses. Entomologia y Vectores 7: 33-47.

Barros, V. L; J. M. M. Rebêlo \& F. S. Silva. 2000. Flebotomíneos (Diptera, Psychodidae) de capoeira do município do Paço do Lumiar, Estado do Maranhão, Brasil. Área endêmica de leishmanioses. Cadernos de Saúde Pública 16: 265-270.

Carvalho, M. L; J. M. M. Rebêlo; J. C. Araújo \& V. L. L. Barros. 2000. Aspectos ecológicos dos flebotomíneos (Diptera, Psychodidae) do município de São José de Ribamar, MA, Brasil. Área endêmica de leishmanioses. Entomologia y Vectores 7: 19-32.

Costa, C. H. N.; H. F. Pereira \& M. Araújo. 1990. Epidemia de Leishmaniose Visceral no estado do Piauí, Brasil. Revista de Saúde Pública 24: 361-372.

Feitosa, M. A. C. \& E. G. Castellón. 2004. Fauna de flebotomíneos (Diptera: Psychodidae) em fragmentos florestais ao redor de conjuntos habitacionais na cidade de Manaus, Amazonas, Brasil. II. Estratificação horizontal. Acta Amazônica 34: 121-127.

Gomes, A. C. \& E. A. B. Galati. 1989. Aspectos ecológicos da leishmaniose tegumentar americana. 7. Capacidade vetorial flebotomínica em ambiente florestal primário do sistema da Serra do Mar, Região do Vale do Ribeira, Estado de São Paulo, Brasil. Revista de Saúde Pública 23: 136-42.

IPPC - Instituto de Pesquisa e Planificação da Cidade. São Luís: uma leitura da cidade. Secretaria Municipal de Planejamento e Desenvolvimento, São Luís, 2006. 94 p.

Laroca, S. 1995. Ecologia: Princípios e Métodos. Petrópolis. Ed. Vozes. 197 p.

Leonardo, F. S. \& J. M. M. Rebêlo. 2004. A periurbanização de Lutzomyia whitmani em área de foco de leishmaniose cutânea, no Estado do Maranhão, Brasil. Revista da Sociedade Brasileira de Medicina Tropical 37: 282-284.

Rebêlo, J. M. M. 2001. Frequiência horária e sazonalidade de Lutzomyia longipalpis (Diptera: Psychodidae: Phlebotominae) na ilha de São Luís, Maranhão, Brasil, Cadernos de Saúde Pública 17: 221 227.

Rebêlo, J. M. M; J. C. Araújo; M. L. Carvalho; V. L. L. Barros; F. S. Silva \& S. T. Oliveira. 1999a. Flebótomos (Lutzomyia, Phlebotominae) da ilha de São Luís, zona do Golfão maranhense, Brasil. Revista da Sociedade Brasileira de Medicina Tropical 32: 247-253

Rebelo, J. M. M.; F. S. Leonardo; J. M. L. Costa; Y. N. O. Pereira \& F. S. Silva. 1999b. Flebotomíneos (Diptera, Psychodidae) de área endêmica de leishmanioses na região dos cerrados, estado do Maranhão, Brasil. Cadernos Saúde Pública 15: 623-630.

Rebêlo, J. M. M; S. T. Oliveira; V. L. L. Barros \& F. S. Silva. 2000a. Flebotomíneos (Diptera, Psychodidae) de Lagoas, município de Buriticupu, Amazônia maranhense. I - Riqueza e abundância relativa das espécies em área de colonização recente. Revista da Sociedade Brasileira de Medicina Tropical 33: 11-19.

Rebêlo, J. M. M.; S. T. Oliveira; V. L. L. Barros \& F. S. Silva. 2000b. Flebotomíneos da Amazônia maranhense. IV - Riqueza e abundância relativa das espécies em área de colonização antiga. Entomologia y Vectores 7: 61-72.

Ryan, L.; R. Lainson; J. J. Shaw \& H. Fraiha Neto. 1987. Ecologia de flebotomíneos (Diptera, Psychodidae, Phlebotominae) na região amazônica. Instituto Evandro Chagas "50 anos", Belém, 1: $307-320$.

Shaw, J. J. \& R. Lainson. 1972. Leishmaniasis in Brazil: IV. Observations on the seasonal variation of Lutzomyia flaviscutellata in different types of forest and its relationship to enzootic rodent leishmaniasis (Leishmania mexicana amazonensis). Transactions of the Royal Society of Tropical Medicine and Hygienne 66: 709-717.

Silveira Neto, S.; O. Nakano; D. Barbin \& N. A. Villa Nova. 1976. Manual de ecologia dos insetos. Agronômica Ceres. São Paulo. $419 \mathrm{p}$.

Ward, R. D.; J. J. Shaw; R. Lainson \& H. Frahia. 1973. Leishmaniasis in Brazil: VIII. Observations on the Phlebotomine fauna of na area highly endemic for cutaneous leishmaniasis in the Serra dos Carajás, Pará State. Transactions of the Royal Society of Tropical Medicine and Hygienne 67: 174-183.

Young, D. G. \& M. A. Duncan. 1994. Guide to the identification and geographic distribution of Lutzomyia sand flies in Mexico, the West Indies; Central and South America (Diptera: Psychodidae). Memories of the American Entomology Institute 54: 1-881.

Recebido em 17/07/2007; aceito em 10/01/2008 